\title{
Exclusive Disjunction and the Biconditional: An Even-Odd Relationship
}

\author{
JOSEPH S. FULDA \\ Mount Sinai School of Medicine \\ New York, NY 10029-6574
}

An elementary truth table argument shows that exclusive disjunction is just the negation of the biconditional: $(P \oplus Q) \equiv \neg(P \Leftrightarrow Q)$. This relationship is sometimes used to explain why inclusive, rather than exclusive, disjunction is the standard disjunction. Either disjunction can be formed from the other $((P \vee Q) \equiv((P \oplus Q) \oplus$ $(P \wedge Q)) ;(P \oplus Q) \equiv((P \vee Q) \wedge \neg(P \wedge Q)))$, but only exclusive disjunction is the negation of another simple connective.

However, while $P \oplus Q$ is logically equivalent to the negation of $P \Leftrightarrow Q, P \oplus Q \oplus R$ is logically equivalent to $P \Leftrightarrow Q \Leftrightarrow R$ itself. (One can omit all parentheses in logical expressions involving only $\oplus$ or $\Leftrightarrow$, since both connectives are commutative and associative.) The reason for this is that $\oplus$ is a mutual exclusivity connective, whereas $\Leftrightarrow$ is an identity connective. Hence, $P \oplus Q \oplus R$ is true precisely when $P \oplus Q$ and $R$ have opposite truth values, which occurs precisely when $P \Leftrightarrow Q$ and $R$ have identical truth values. Generalizing this pattern gives strings of propositions connected by $\oplus$ or $\Leftrightarrow$ that alternate in accordance with the following identities:

$$
\begin{aligned}
& \text { (A) } \bigoplus_{i=1}^{n} P_{i} \equiv \stackrel{n}{\Leftrightarrow} P_{i}, \quad \text { for } n \text { odd; } \\
& \text { (B) } \bigoplus_{i=1}^{n} P_{i} \equiv \neg\left(\underset{i=1}{\stackrel{n}{\Leftrightarrow}} P_{i}\right), \quad \text { for } n \text { even. }
\end{aligned}
$$

We now prove these identities by mathematical induction on the number of propositions.

Proof. Basis: The logical equivalence $P_{1} \oplus P_{2} \equiv \neg\left(P_{1} \Leftrightarrow P_{2}\right)$ follows directly from the truth tables for the two expressions.

Induction Step: Assume the identities true for an integer $n \geq 2$. We will show them true for $n+1$.

(A) $n$ is odd. We begin with $\oplus_{i=1}^{n+1} P_{i}$, which can be rewritten $\left(\oplus_{i=1}^{n} P_{i}\right) \oplus P_{n+1}$. By the basis, this is equivalent to $\neg\left(\left(\oplus_{i=1}^{n} P_{i}\right) \Leftrightarrow P_{n+1}\right)$. By the induction hypothesis, this is equivalent to $\neg\left(\left(\Leftrightarrow{ }_{i=1}^{n} P_{i}\right) \Leftrightarrow P_{n+1}\right)$. This, in turn, is just $\neg\left(\Leftrightarrow{ }_{i=1}^{n+1} P_{i}\right)$, which concludes the induction step for case (A) and with it the proof of case (A).

(B) $n$ is even. We begin with $\oplus_{i=1}^{n+1} P_{i}$, which can be rewritten $\left(\oplus_{i=1}^{n} P_{i}\right) \oplus P_{n+1}$. By the basis, this is equivalent to $\neg\left(\left(\oplus_{i=1}^{n} P_{i}\right) \Leftrightarrow P_{n+1}\right)$. By the induction hypothesis, this is equivalent to $\neg\left(\neg\left(\Leftrightarrow{ }_{i=1}^{n} P_{i}\right) \Leftrightarrow P_{n+1}\right)$. Since $\neg(\neg P \Leftrightarrow Q)$ is true just when $P$ and $Q$ have identical truth values (i.e., $\neg(\neg P \Leftrightarrow Q) \equiv(P \Leftrightarrow Q))$, this in turn yields $\left(\Leftrightarrow{ }_{i=1}^{n} P_{i}\right) \Leftrightarrow P_{n+1}$, which is just $\Leftrightarrow{ }_{i=1}^{n+1} P_{i}$. This concludes the induction step for case (B) and with it the proof of case (B).

Acknowledgments. I would like to acknowledge the comments of an anonymous referee. I also wish to dedicate this note to Mr. Samuel Block, an outstanding teacher of mathematics, from whom I first learned to appreciate identities and their proofs. 